\title{
AN EXPERIMENTAL MANIPULATION OF LIFE-HISTORY TRAJECTORIES AND RESISTANCE TO OXIDATIVE STRESS
}

\author{
Carlos Alonso-Alvarez,,${ }^{1,2}$ Sophie Bertrand, ${ }^{1}$ Godefroy Devevey,,${ }^{3,4}$ Josiane Prost, ${ }^{5}$ Bruno Faivre, ${ }^{3}$ \\ Olivier Chastel, ${ }^{6}$ and Gabriele Sorci ${ }^{1,7}$ \\ ${ }^{1}$ Laboratoire de Parasitologie Evolutive, Université Pierre et Marie Curie, Centre National de la Recherche Scientifique, \\ Unite Mixte de Recherche, 7103, 7 quai St. Bernard, 75252 Paris cedex 05, France \\ ${ }^{3}$ Université de Bourgogne, Centre National de la Recherche Scientifique, Unite Mixte de Recherche, 5561 BioGéoSciences, \\ Equipe Ecologie Evolutive, 6 Blvd Gabriel, 21000 Dijon, France \\ ${ }^{5}$ Université de Bourgogne, Unité Propre de Recherche de l'Enseignement Supérieur Lipides Nutrition, EA 2422, 6 Blvd Gabriel, \\ 21000 Dijon, France \\ ${ }^{6}$ Centre d'Etudes Biologiques de Chizé-Centre National de la Recherche Scientifique, 79360 Villiers en Bois, France
}

\begin{abstract}
Optimal investment into life-history traits depends on the environmental conditions that organisms are likely to experience during their life. Evolutionary theory tells us that optimal investment in reproduction versus maintenance is likely to shape the pattern of age-associated decline in performance, also known as aging. The currency that is traded against different vital functions is, however, still debated. Here, we took advantage of a phenotypic manipulation of individual quality in early life to explore (1) long-term consequences on life-history trajectories, and (2) the possible physiological mechanism underlying the life-history adjustments. We manipulated phenotypic quality of a cohort of captive zebra finches (Taeniopygia guttata) by assigning breeding pairs to either an enlarged or a reduced brood. Nestlings raised in enlarged broods were in poorer condition than nestlings raised in reduced broods. Interestingly, the effect of environmental conditions experienced during early life extended to the age at first reproduction. Birds from enlarged broods delayed reproduction. Birds that delayed reproduction produced less offspring but lived longer, although neither fecundity nor longevity were directly affected by the experimental brood size. Using the framework of the life-table response experiment modeling, we also explored the effect of early environmental condition on population growth rate and aging. Birds raised in reduced broods tended to have a higher population growth rate, and a steeper decrease of reproductive value with age than birds reared in enlarged broods. Metabolic resources necessary to fight off the damaging effect of reactive oxygen species (ROS) could be the mechanism underlying the observed results, as (1) birds that engaged in a higher number of breeding events had a weaker red blood cell resistance to oxidative stress, (2) red blood cell resistance to oxidative stress predicted short-term mortality (but not longevity), and (3) was related with a parabolic function to age. Overall, these results highlight that early condition can have long-term effects on life-history trajectories by affecting key life-history traits such as age at first reproduction, and suggest that the trade-off between reproduction and self-maintenance might be mediated by the cumulative deleterious effect of ROS.
\end{abstract}

Key words._-Age at first reproduction, disposable-soma theory of aging, free radicals, life-table response experiment, resistance to oxidative stress, senescence.

Received November 15, 2005. Accepted June 19, 2006.

One of the central tenets of evolutionary biology is that life-history traits cannot evolve independently from one another but instead are embedded in a matrix of correlations (Stearns 1992). These correlations are known as trade-offs and reveal the constraints that shape the evolution of fitnesslinked traits (Reznick 1992; Rose and Bradley 1998; Zera and Harshman 2001). One of the most generally accepted hypotheses explaining why life-history traits are usually negatively correlated refers to the allocation of limited resources to conflicting functions. In this context, organisms have to face a decision about the optimal allocation of metabolic resources between self-maintenance and reproduction. In theory, maximal investment into self-maintenance should con-

\footnotetext{
2 Present address: Instituto de Investigación en Recursos Cinegéticos, (CSIC, UCLM, JCCM), Ronda de Toledo s/n, 13005Ciudad Real, Spain; E-mail: carlos.alonso@uclm.es.

${ }^{4}$ Present address: Département d'Ecologie et Evolution, Université de Lausanne, Bâtiment de Biologie, Dorigny, 1015 Lausanne, Switzerland.

7 Present address: Université de Bourgogne, Centre National de la Recherche Scientifique, Unite Mixte de Recherche, 5561 BioGéoSciences, Equipe Ecologie Evolutive, 6 Blvd Gabriel, 21000 Dijon, France.
}

siderably delay the progressive impairment of functions, also known as aging, at the expense of progeny production. But a maximal investment into self-maintenance might be a bad option if sources of extrinsic mortality (e.g., predation, starvation, infectious diseases) would never allow individuals to express such an extended longevity. This reasoning is at the base of the disposable-soma theory of the evolution of aging (Kirkwood 1977; Kirkwood and Rose 1991) that predicts that the optimal solution to the resource allocation dilemma is to invest fewer resources to the maintenance of somatic structures than are required for extended lifespan (e.g., Carranza et al. 2004). Although the disposable-soma theory provides an evolutionary explanation for the aging process, at the proximal level, the mechanisms linking self-maintenance and reproduction are poorly understood, and particularly the nature of the resources traded between different functions is still debated (Kirkwood 2005). It has been suggested that the cause of the age-associated decline in performance could be the accumulation of damages produced by reactive oxygen species (ROS) throughout life ("free-radical theory", of aging; Harman 1957). ROS are unstable and very reactive byproducts of normal metabolic activities, presenting damaging effects on the principal biomolecules such as DNA, proteins, 
and lipids (Finkel and Holbrook 2000). To temper the negative effects of ROS, aerobic organisms have evolved sophisticated antioxidant defenses based on compounds which scavenge and quench ROS. The imbalance between the production of ROS and antioxidant molecules is commonly defined as oxidative stress (Finkel and Holbrook 2000). According to the disposable soma theory and our current knowledge on oxidative stress, aging would thus arise as a consequence of limited investment in antioxidant defenses at older ages.

In this paper we aim to test the predictions of the disposable-soma theory of aging in a nonmammalian vertebrate species: the zebra finch (Taeniopygia guttata). Using an experimental approach, we manipulated early phenotypic condition for a cohort of birds. Beyond any genetic mechanism underlying the correlated evolution of life-history traits, it is now well established that environmental conditions experienced during the early stages of an organism can have a substantial impact on adult traits (Lindström 1999; Lumma and Clutton-Brock 2002; Bateson et al. 2004; Ozanne and Hales 2004; Gluckman et al. 2005). We manipulated early condition of zebra finches by experimentally allowing parents to raise a brood of either two or six chicks. Afterwards, we followed the life-history trajectory of the cohort of individuals produced under these two regimes. We monitored a number of physiological variables supposed to reflect aspects of individual quality (resistance of red blood cells to a controlled free-radical attack, immune response, plasma concentration of carotenoids and total protein, body mass, and size) at different ages, and the expression of life-history traits (age at first reproduction, reproductive output, and longevity).

\section{Materials AND Methods}

\section{General Procedure}

The study was conducted on captive zebra finches. Randomly formed pairs were placed in breeding cages $(0.6 \times$ $0.4 \times 0.4 \mathrm{~m}$ ) with food (a commercial mix of seeds) and water ad libitum. This was the first breeding attempt for all birds (all less than one year old). Birds were obtained during October 2002 from two different French pet providers. The breeding cages were maintained in a room with constant temperature $\left(21^{\circ} \pm 1^{\circ} \mathrm{C}\right)$ and light-dark daily cycles (13L: 11D). Each breeding pair was randomly allocated into one of two groups and allowed to raise either a two-chick or a six-chick brood. Thirty-four pairs reared reduced broods and 18 pairs reared enlarged broods. To disrupt the natural covariation between brood size and parental quality, brood size was manipulated when nestlings were two-days old. Initial clutch size did not differ between the groups that further received two or six chicks (mean \pm SE: $4.21 \pm 0.21$ and $4.67 \pm 0.67$, respectively; $\left.F_{1,50}=1.20, P=0.277\right)$. Hatchlings were randomly allocated to synchronous broods. Chicks were housed with their rearing parents (original or foster parents) until they were 40 days old. Afterwards, the cage was divided into two compartments; chicks were physically separated from their parents but remained in visual and acoustic contact with them. Both halves of the cage were provided with food and water ad libitum. Chicks were maintained in the cages until they were 60 days old, when they were released into a large outdoor aviary $(18 \mathrm{~m}$ length $\times 3 \mathrm{~m}$ wide $\times 2.5 \mathrm{~m}$ high $)$ with water and food ad libitum. Boiled hen eggs and lettuce were also provided once a week. The aviary contained 90 nestboxes and nesting material (hay) for breeding. A total of 140 birds were released between April 5 and July 3, 2003 (reduced broods: 26 males and 36 females; enlarged broods: 37 males and 41 females; $\left.\chi^{2}=0.422, P=0.516\right)$. Each individual wore a unique combination of color rings for further identification. Because red rings have been shown to modify sexual attractiveness in zebra finches (Burley 1988), we avoided using this color. Birds were recaptured in the outdoor aviary at three occasions, on September 19, 2003, and March 25 and May 26, 2004. Birds were not captured more frequently to limit disturbances. Individuals born in the outdoor aviary were removed at each of these capture events.

\section{Assessing Individual Quality}

Tarsus length ( $\pm 0.05 \mathrm{~mm}$ ) was measured when nestlings were 20 and 60 days old. Body mass ( $\pm 0.1 \mathrm{~g}$ ) was assessed at the age of 20 and 60 days, and again when birds were recaptured in the outdoor aviary. A blood sample was also taken at each of these handling events. To determine the change in the plasma concentration of carotenoids and total protein, the first and second blood samples were centrifuged and plasma was stored at $-20^{\circ} \mathrm{C}$.

The delayed hypersensitivity assay (Goto et al. 1978) was used to evaluate the in vivo T-cell mediated immune response, following the procedure described in Smits et al. (1999; see also Alonso-Alvarez and Tella 2001). When 20 days old, birds were injected subcutaneously in the right wing web with $50 \mu \mathrm{g}$ of phytohemagglutinin (PHA; SIGMA, St.Louis, MO) diluted in $20 \mu \mathrm{l}$ of phosphate buffered saline (PBS). The thickness of the wing web was measured at the injection site just prior to and $24 \mathrm{~h}$ after challenge using a spessimeter. The wing web swelling was calculated as the difference between the thickness of the wing web prior to and $24 \mathrm{~h}$ after injection.

In addition to body mass and size, we assessed total protein level, plasma carotenoids, and cell-mediated immune response to have a wider picture of the effect of brood size manipulation on individual quality because total protein and immune response might reflect nutritional status (Brown 1996; Gonzalez et al. 1999; Alonso-Alvarez and Tella 2001; but see e.g., Dawson and Bortolotti 1997) and carotenoids might reflect the availability of antioxidant defenses (von Schantz et al. 1999; Alonso-Alvarez et al. 2004a).

\section{Measurement of Total Carotenoid Concentration in Plasma}

Twenty $\mu \mathrm{l}$ of plasma were diluted in $180 \mu \mathrm{l}$ of absolute ethanol. The dilution was mixed in a vortex and the flocculent protein was precipitated by centrifuging the sample at 1500 $\mathrm{g}$ for 10 minutes. We examined the supernatant in a spectrophotometer and determined the optical density of the carotenoid peak at $450 \mathrm{~nm}$. Carotenoid concentration was determined from a standard curve of lutein. We have previously shown that values obtained with this method are highly correlated with the total amount of carotenoids determined by HPLC (Alonso-Alvarez et al. 2004a). 


\section{Measurement of Plasma Total Protein}

Total protein present in the plasma was determined by the Biuret method, using an automatic spectrophotometer (Technicon RA 1000, Technicon, Saint-Denis, France). All samples were analyzed in the same time series.

\section{Measurement of Red Blood Cell Resistance to Oxidative Stress}

Resistance to oxidative stress was assessed as the time needed to hemolyze $50 \%$ of red blood cells exposed to a controlled free-radical attack. We used the KRL test (Brevet Spiral V02023, Couternon, France; http://www.nutriteck. com/sunyatakrl.html) adapted to avian physiological parameters (Alonso-Alvarez et al. 2004a,b; Bertrand et al. 2006).

At each blood sampling date, $20 \mu \mathrm{l}$ of whole blood were immediately diluted in $730 \mu \mathrm{l}$ of KRL buffer $\left(150 \mathrm{mM} \mathrm{Na}{ }^{+}\right.$, $120 \mathrm{mM} \mathrm{Cl}^{-}, 6 \mathrm{mM} \mathrm{K}^{+}, 24 \mathrm{mM} \mathrm{HCO}_{3}^{-}, 2 \mathrm{mM} \mathrm{Ca}^{2+}, 340$ mosm, $\mathrm{pH} 7.4$ ) and stored at $4^{\circ} \mathrm{C}$ before analysis that occurred within 24 hours.

Eighty $\mu \mathrm{l}$ of whole blood diluted in the KRL buffer were added to each well of a 96-well microplate. We subsequently added to each well $136 \mu \mathrm{l}$ of a $150 \mathrm{mM}$ solution of $\left[2,2^{\prime}-\right.$ azobis-(amidinopropane)hydrochloride (AAPH)] (646 mg of AAPH diluted in $20 \mathrm{ml}$ of KRL buffer), a free-radical generator (Rojas Wahl et al. 1998). The microplate was subsequently read with a microplate titrator (iEMS Reader MF, Kirial SA, Couternon, France) at $40^{\circ} \mathrm{C}$. The rate of hemolysis is determined by the change in optical density measured at $540 \mathrm{~nm}$. Previous work has shown that, if at least one component of the antiradical detoxification system is impaired, the hemolysis curve shows a shift towards shorter times (Blache and Prost 1992; Girard et al. 2005). This test therefore provides an assessment of resistance to oxidative stress because all families of antioxidants present in the blood are used to fight off the oxidant attack (Blache and Prost 1992; Pieri et al. 1996; Girodon et al. 1997; Lesgards et al. 2002; Stocker et al. 2003; Girard et al. 2005).

\section{Assessing Life-History Trajectories}

Reproductive behavior was monitored from the release in the outdoor aviary until the death of the last individual of a given sex, on June 23, 2004 (nine males were still alive when the last female died). The age at first reproduction, the total number of breeding events (any clutch laid, independently of whether it failed or not), the total number of fledglings produced, and the longevity (number of days alive) were determined for each individual. Nest-boxes were checked every two days to record any breeding attempt. Parents were identified by recording the nest-box with a digital video camera (Panasonic NV-MX7EG, Osaka, Japan) and by direct observation from incubation to chick-rearing period.

\section{Statistical Analyses}

Data were analyzed using mixed models and repeated-measurements mixed models (PROC MIXED in SAS statistical software; SAS Institute 2001). To control for the effect of shared genes and environment, data were cross-classified by introducing the original and foster nests as random factors.
Moreover, the identity of each bird was included as a third random factor in the repeated-measurements mixed models.

When the dependent variables were not normally distributed (i.e., clutch size and number of fledglings per breeding event), we used generalized linear mixed models (PROC GLIMMIX in SAS; SAS Institute 2001). This allowed us to choose the appropriate error distribution and link function (Poisson error and log link). Again, nest of origin, nest of rearing and identity of each bird were added as random effects.

Experimental brood size (two vs. six chicks) and sex were included as fixed factors in each model. To account for a possible influence of environmental conditions at the time of release, the date of release into the outdoor aviary was also added but was never significant (always $P>0.05$ ). Similarly, to take into account that birds were either raised in their original or in a foster nest, a two-level factor (original vs. foster parents) was included in all the models. This factor, as well as the two-way interactions with the other variables, never reached the significance threshold $(P>0.40)$. Satterthwaite correction was used to approximate the degrees of freedom (Littell et al. 1996).

Survival curves were analyzed with log-rank tests (PROC LIFETEST) and Cox regressions (PROC PHREG, both in SAS software; SAS Institute 2001).

\section{Life Table Response Experiment Modeling}

In addition to the models based on individual values, we also used the life table response experiment (LTRE) modeling to assess the effect of early condition on life-history trajectories and aging. In LTRE, a treatment is applied that is supposed to affect vital rates in an age-specific manner (Caswell 2001). Matrix models allow one to identify how a treatment affects population growth rate $(\lambda)$, how age-specific population parameters contribute to the effect of the treatment on $\lambda$, and to synthesize these effects into statistics, such as the reproductive value.

Age-specific survivorship and fertility of females were computed for each of eight age classes (the age classes were based on a 50-day interval, starting from the day of release in the outdoor aviary) for the two experimental groups. The 50-day interval was chosen because it corresponds to the average time period between two consecutive breeding events (laying-nestling independence; Zann 1996). Life tables were used to construct age-classified population projection matrices (Leslie matrices) (A $\mathbf{A}^{\text {red }}$ and $\mathbf{A}^{\text {enl }}$ for females reared in reduced and enlarged broods, respectively).

Population growth rate $(\lambda)$ was calculated as the dominant eigenvalue of each matrix, and the reproductive value $(v)$ as the left eigenvector (Caswell 2001). The contribution matrix (the contribution of demographic variables to observed changes in $\lambda$ due to the treatment) was calculated as (Caswell 2001):

$$
\mathbf{C}=\mathbf{D} \text { o } \mathrm{S}_{\mathbf{A}}^{\dagger}
$$

where $\mathbf{D}$ is the matrix of differences

$$
\mathbf{D}=\mathbf{A}^{\mathrm{red}}-\mathbf{A}^{\mathrm{enl}}
$$

and $\mathrm{S}_{\mathrm{A}}$ is the sensitivity of the average matrix 


$$
\mathbf{A}^{\dagger}=\left(\mathbf{A}^{\text {red }}+\mathbf{A}^{\text {enl }}\right) / 2
$$

The statistical significance of brood size-induced differences in demographic parameters was assessed using a randomization test (Levin et al. 1996; Caswell 2001). Population growth rates, contributions to $\lambda$, and reproductive values were recalculated by permuting individuals between treatments, keeping the original sample size for each treatment. For each permutation, a new projection matrix was built and $\lambda$, agespecific contributions to $\lambda$, and reproductive values, were computed. We performed 500 permutations providing 500 re-sampled demographic values. To assess the statistical significance of $\lambda$ we computed the difference between the 500 simulated population growth rates $\left(\lambda^{\text {red }}-\lambda^{\text {enl }}\right)$, and looked at the location of the observed difference within the distribution of simulated differences (Levin et al. 1996; Caswell 2001). Observed values larger or smaller than the upper $95 \%$ or lower $5 \%$ percentile of the simulated distribution were considered as significant. Similarly, statistical significance of age-specific contributions to $\lambda$ was approached by comparing the observed values with the upper $95 \%$ and lower $5 \%$ percentile of the simulated distribution. For the reproductive value, we computed the difference $\left(v_{d}=v_{\text {red }}-v_{\text {enl }}\right)$ for each age class, regressed these differences on age and estimated the slope of the regression. To test whether the observed slope could have been generated as a consequence of a random process, we computed the differences in reproductive values for each permutated dataset and computed the slope between the differences in reproductive values and age. In this way, we generated a set of 500 randomly generated slopes, and as previously described, we compared the observed slope with the distribution of the simulated slopes.

\section{RESUlTS}

\section{Individual Quality}

Birds reared in enlarged broods had smaller tarsi and lower body mass than nestlings in reduced broods when they were 20 and 60 days old (Figs. 1A and B). However, the difference between the two groups tended to decrease at 60 days of age which resulted in a significant brood size by age interaction for both tarsus length and body mass (Table 1).

Plasma protein increased between the two sampling ages ( 20 vs. 60 days old), however, neither the brood size nor the sex affected the total amount of plasma protein (Table 1).

Brood size had a strong effect on variation in plasma carotenoids. Birds reared in enlarged broods tended to have smaller amounts of plasma carotenoids when 20 days old compared to chicks raised in reduced broods, and this difference became much stronger at 60 days of age (Fig. 1C). This resulted in a significant brood size by age interaction (Table 1).

Brood size also affected the intensity of the cell-mediated immune response as assessed at 20 days of age and this effect was independent of body mass (brood size: $F_{1,44.6}=11.9$, $P=0.001$; sex: $F_{1,97.7}=0.30, P=0.583$; body mass: $F_{1,128}$ $=0.19, P=0.664$; two-chick brood, mean $\pm \mathrm{SE}=1.16 \pm$ $0.05 \mathrm{~mm}$, six-chick brood, mean $\pm \mathrm{SE}=0.87 \pm 0.06 \mathrm{~mm}$ ).

Finally, resistance of red blood cells to a controlled freeradical attack at 60 days of age was significantly weaker for
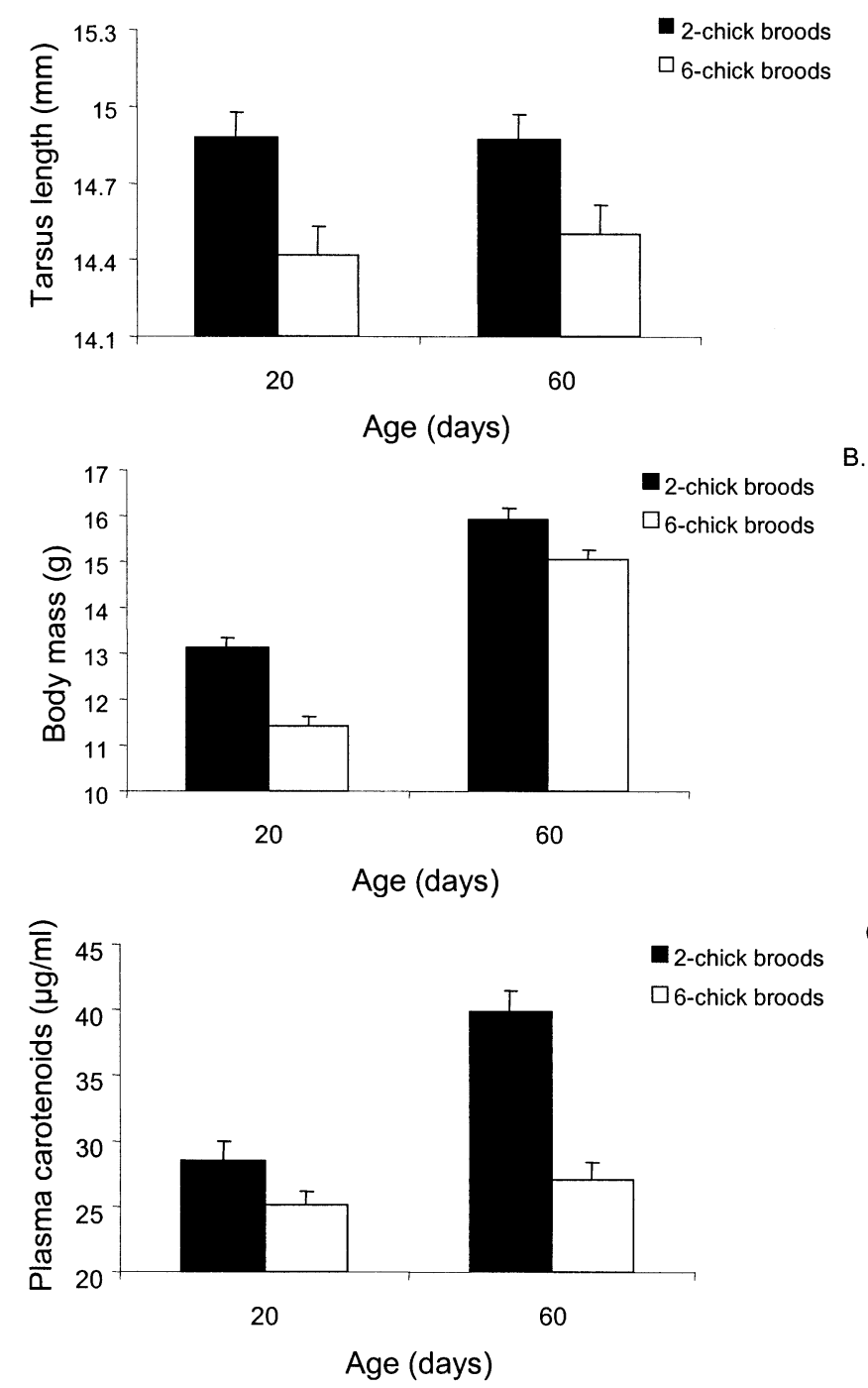

FIG. 1. Tarsus length (A), body mass (B), and carotenoid plasma levels (C) of zebra finches reared in experimentally reduced or enlarged broods as a function of age (20 and 60 days old). Bars represent least-square means $\pm \mathrm{SE}$ from repeated mixed models.

birds in enlarged broods (brood size: $F_{1,38.6}=4.99, P=$ 0.031 ; sex: $F_{1,105}=0.19, P=0.661$; body mass: $F_{1,101}=$ $3.82, P=0.053$; two-chick brood, mean $\pm \mathrm{SE}=61.83 \pm$ $0.63 \mathrm{~min}$, six-chick brood, mean $\pm \mathrm{SE}=59.79 \pm 0.67 \mathrm{~min})$.

\section{Reproductive Traits}

Seven birds were not observed to breed, five of which were from enlarged broods. Most birds reproduced when they were three months old (median, min-max $=94$, 66-321 days). Lifetime reproductive success was very skewed with relatively few birds contributing to most of the offspring produced [median number of clutches $=5(\min -\max =1-13)$; median number of fledglings $=4(\min -\max =0-18)]$. Median lifespan was 332 days (min-max, 62-505 days).

Age at first reproduction was significantly earlier for individuals coming from reduced broods (Table 2; Fig. 2). Birds that started to breed earlier had higher number of total 
LIFE HISTORIES AND OXIDATIVE STRESS

reproductive attempts over their lifespan (slope $\pm \mathrm{SE}=$ $-4.58 \pm 1.29$; Table 2 ). Lifetime reproductive success, assessed as the number of fledglings, was also significantly correlated with age at first reproduction. Birds that started to reproduce earlier produced more fledglings (slope $\pm \mathrm{SE}=$ $-2.02 \pm 0.98$; Table 2).

Birds reared in reduced broods tended to have a higher number of reproductive attempts than birds raised in enlarged broods, although the difference was not statistically significant (mean \pm SE: $5.06 \pm 0.36$ and $4.30 \pm 0.33$, respectively, $F_{1,84.9}=2.51, P=0.117$ ), whereas birds reared in reduced broods produced more fledglings during their lifetime than birds raised in enlarged broods (mean \pm SE: $4.81 \pm 0.55$ and $3.12 \pm 0.66, F_{1,21.7}=4.73, P=0.041$ ). Nevertheless, when including age at first reproduction as a covariate in the model, brood size did not affect the number of reproductive attempts nor the number of fledglings produced (Table 2).

Clutch size showed a slow linear decrease with age $\left(F_{1,288}\right.$ $=4.82, P=0.029$, slope $\pm \mathrm{SE}=-0.001 \pm 0.0003)$; there was no difference in the pattern of decrease between birds reared in enlarged or reduced broods (age $\times$ brood size interaction; $\left.F_{1,280}=0.16, P=0.693\right)$. The number of fledglings in each reproductive event also linearly decreased with age $\left(F_{1,141}=6.69, P=0.011\right.$, slope $\left.\pm \mathrm{SE}=-0.0019 \pm 0.0007\right)$, and the slopes did not differ between birds reared in enlarged or reduced broods (age \pm brood size interaction: $F_{1,144}=$ $0.26, P=0.609)$.

\section{Longevity}

Age at first reproduction predicted longevity, birds that started to breed earlier having shorter lifespan (Cox regression: $\chi^{2}=4.49, P=0.034$; Fig. 3 ). Neither the experimental brood size (Log-rank test $=0.64, P=0.423$ ) nor the sex (Log-rank test $=2.12, P=0.145)$ affected longevity.

\section{Age-Associated Changes in the Resistance to Oxidative Stress}

Resistance to oxidative stress varied with age following a parabolic function (Fig. 4). The pattern of age-associated variation differed for the two brood size groups, as shown by the significant brood size by age and brood size by age squared interactions (brood size: $F_{1,211}=7.85, P=0.006$; sex: $F_{1,106}=1.26, P=0.263$; age: $F_{1,203}=19.65, P<$ 0.0001; age squared: $F_{1,226}=27.28, P<0.0001$; brood size by age: $F_{1,203}=10.36, P=0.0015$; brood size by age squared: $\left.F_{1,224}=8.07, P=0.0049\right)$. Thus, whereas birds from reduced broods showed higher resistance to oxidative stress when 60 days old, the pattern was reversed at the following sampling age (Fig. 4).

\section{Reproductive Effort and Resistance to Oxidative Stress}

The effect of breeding effort on red blood cell resistance to oxidative stress was assessed by running a model with the three breeding variables (age at first reproduction, number of total breeding attempts and number of fledglings) plus the experimental brood size and sex. We used a backward stepwise procedure to find the model with the best fit. This analysis was restricted to the individuals sampled on September 
TABLE 2. Effect of the experimental brood size (two vs. six chicks) on reproductive traits in zebra finches. The values refer to mixed models (see Materials and Methods for more details).

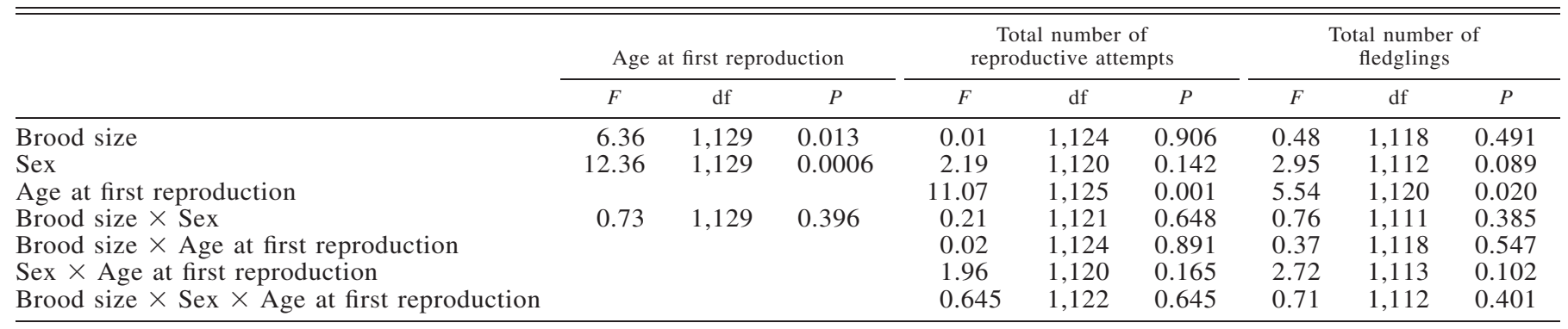

19, 2003 (i.e., the first handling event after bird release in the aviary), because the mortality rate between September and the end of March substantially reduced the sample size and prevented reliable estimates of the effect of breeding effort on resistance to oxidative stress. Sixty-six per cent of the total number of reproductive attempts (i.e., 208/313) occurred in the period between the release in the aviary and September 19, 2003, with significantly more breeding events involving birds from reduced broods than from enlarged broods $\left(F_{1,50}=7.64, P=0.008\right)$. Among the breeding variables, the number of reproductive attempts was the best predictor of resistance to oxidative stress: birds that overall laid more clutches showed the weakest resistance $\left(F_{1,117}=7.77\right.$, $P=0.0062$, slope $\pm \mathrm{SE}=-0.97 \pm 0.35)$. The sex of the bird was also retained in the model, males showing higher resistance to oxidative stress than females $\left(F_{1,117}=5.46, P\right.$ $=0.021$, mean \pm SE: $65.6 \pm 0.96$ and $62.7 \pm 0.87 \mathrm{~min}$, for males and females, respectively). Age at first reproduction and number of fledglings produced were removed as nonsignificant terms (both $P>0.08$ ).

\section{Mortality and Resistance to Oxidative Stress}

To assess the correlation between red blood cell resistance to oxidative stress as assessed in September and mortality, we ran a series of generalized linear models (GLIMMIX with binomial distribution and logit link function) based on a twoweek time step. We did this to test whether red blood cell resistance to oxidative stress predicted mortality over a short or long period of time following the measurement. Figure 5 reports the $P$ values of these models and shows that resistance

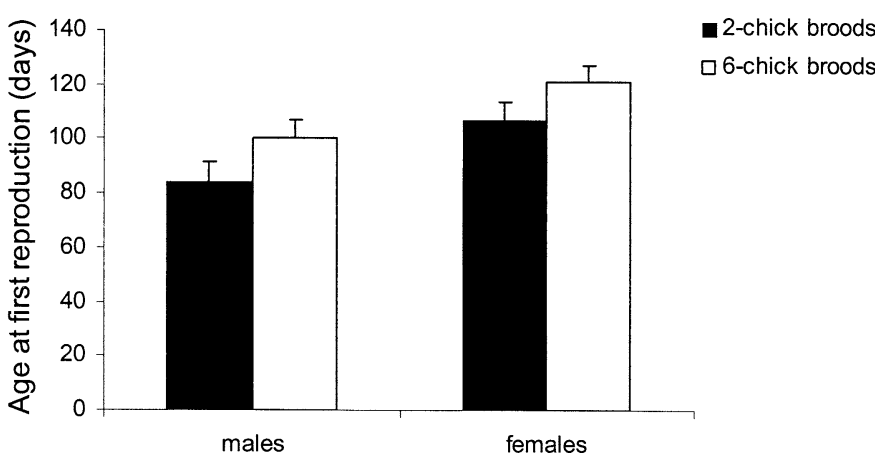

FIG. 2. Age at first reproduction of zebra finches reared in experimentally reduced or enlarged broods. Bars represent leastsquare means \pm SE from a mixed model of covariance. to oxidative stress was associated to mortality up to three months following its measurement. Mortality that occurred after this three months period was random with respect to resistance to oxidative stress. Figure 6 shows the relationship between red blood cell resistance to oxidative stress and mortality during the three months that followed its measurement. One can note that the magnitude of the effect was substantial as the likelihood of dying during this period was about twice as high for birds with a weak resistance compared with birds that exhibited a strong resistance. Finally, red blood cell resistance to oxidative stress as assessed in September was not correlated with lifespan $\left(F_{1,84.4}=0.53, P=0.469\right)$.

\section{Life Table Response Experiment Modeling}

Population growth rate of birds reared in enlarged broods was $\lambda^{\text {enl }}=1.068$ versus $\lambda^{\text {red }}=1.235$ for birds from reduced broods. In other terms, the population of birds from enlarged broods would have increased with a rate of $6.8 \%$ per timeunit, whereas the population of birds from reduced broods would have increased with a rate of $23.5 \%$ per time-unit, a 3.46-fold difference. However, this difference was not sta-

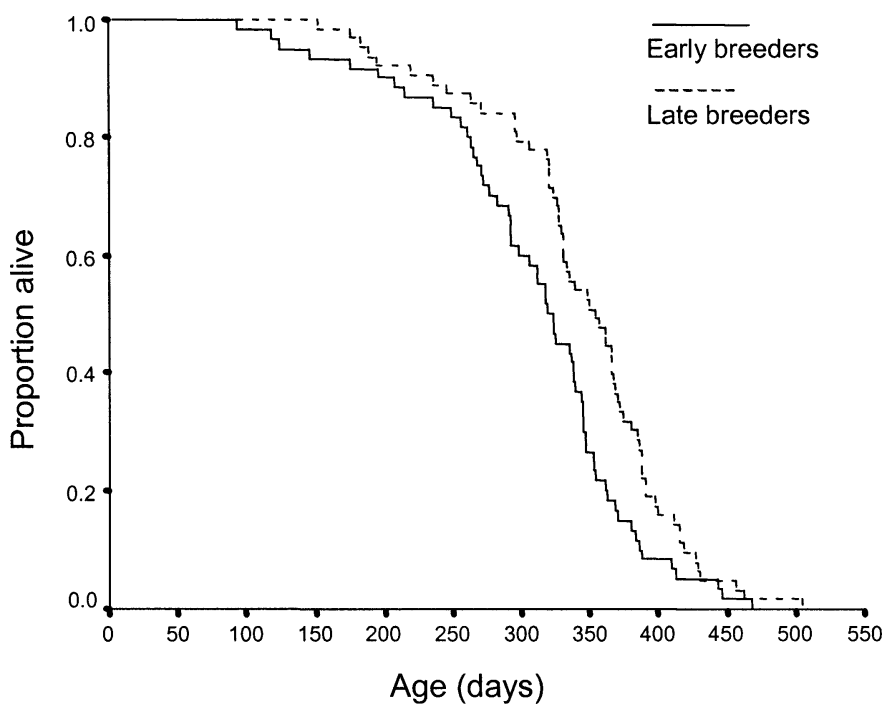

FIG. 3. Survival curves of early and late breeding zebra finches. To illustrate the relationship between age at first reproduction and survival, individuals were scored as early breeders if they reproduced when less than 94 days old (i.e., the median age at first reproduction) or as late breeders if they reproduced when more than 94 days old. 


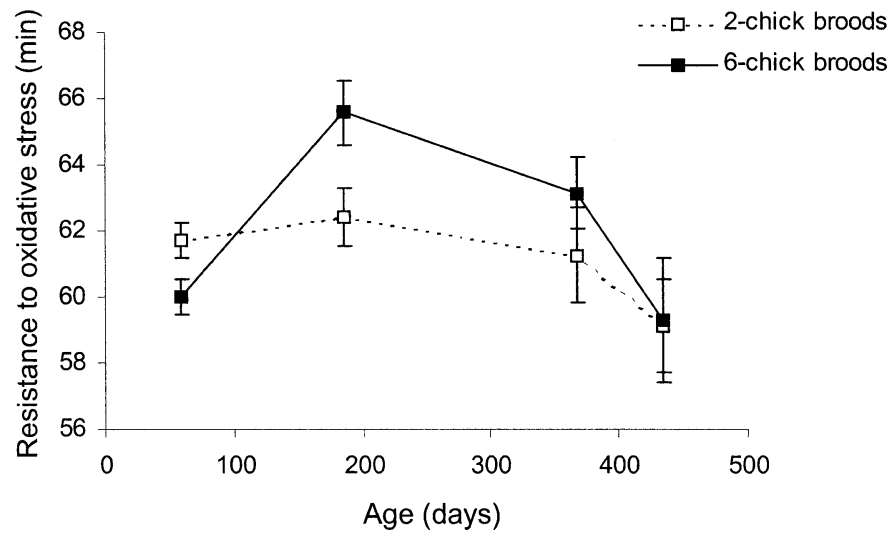

FIG. 4. Age-associated variation in resistance to oxidative stress of zebra finches reared in experimentally reduced or enlarged broods. Resistance to oxidative stress was the time (min) needed to hemolyze $50 \%$ of red blood cells exposed to a controlled freeradical attack. Individuals were sampled when they were 60 days old and again after their release in the outdoor aviary on September 19, 2003; March 25, 2004; and May 26, 2004. At these dates the average age of sampled birds was 186,368 , and 434 days, respectively. Means $\pm \mathrm{SE}$ are reported.

tistically significant as $10 \%$ of the simulated differences were equal to or greater than the observed difference $\left(\lambda^{\text {red }}-\lambda^{\text {enl }}\right.$ $=0.167 ; P=0.100$ ).

Differences in early fecundity between birds from reduced and enlarged broods had the largest contributions to differences in $\lambda$ (not surprisingly, late fecundity had almost no effect on $\lambda$ ) (Fig. 7A). Overall, differences in survival between reduced and enlarged brood individuals had weak contributions to differences in $\lambda$ (Fig. 7B). These results indicate that $\lambda$ was much more sensitive to changes in fecundity than to changes in adult survival. We should, however, keep in mind that that $\lambda$ values did not significantly differ between treatments (see above).

To compare reproductive values of birds from enlarged and reduced broods (Fig. 8A), we computed the differences in reproductive values $\left(v_{d}=v_{\text {red }}-v_{\text {enl }}\right)$ for each age class (Fig. 8B). Differences in reproductive values were positive at early ages (birds from reduced broods having higher reproductive values than birds from enlarged broods) and became negative at older ages (birds from reduced broods having lower reproductive values than birds from enlarged broods). This pattern was best described by a linear negative regression with a standardized slope $=-0.934$ (Fig. 8B). To test whether this negative slope could have arisen as a consequence of a random process, we compared the observed slope to the distribution of 500 randomly generated slopes. Only seven out of the 500 randomly generated slopes were lower than the observed value. Thus, the probability that the observed negative relationship between differences in reproductive values and age would arise as a consequence of a random process was as low as 0.014 .

\section{Discussion}

We found that increased nestling competition, induced by an experimental brood size manipulation, extensively affected a number of physiological parameters, determined the age at first reproduction, and accelerated the decline of reproductive value with age (an index of aging) in a cohort of zebra finches. Birds that experienced good conditions during early development were of better quality as assessed by body mass, tarsus length, immune response, plasma carotenoids, and resistance of red blood cells to a free-radical attack. They also started to breed earlier. Earlier reproduction resulted in a higher number of breeding events and offspring produced

\section{Number of weeks after the assessment of red blood cell resistance to oxidative stress}

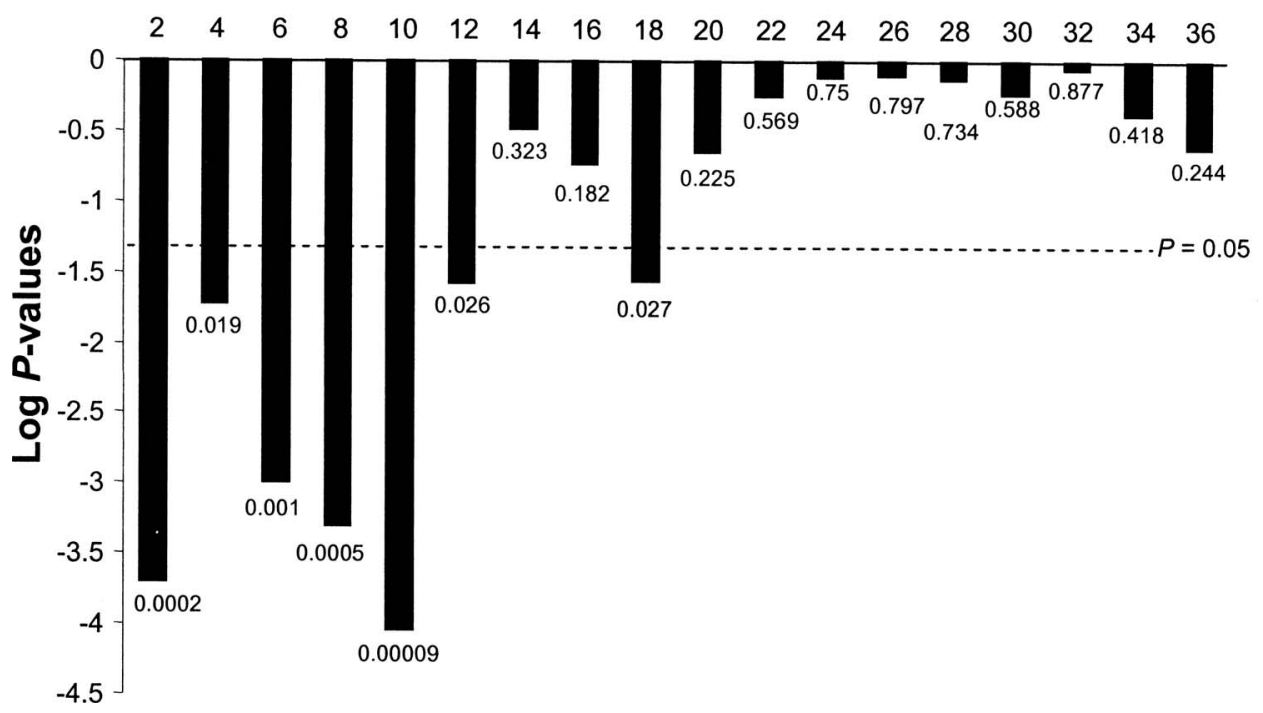

FIG. 5. Distribution of $\log _{10}$-transformed $P$-values from generalized linear models exploring the association between red blood cell resistance to oxidative stress and mortality. Each $P$-value corresponds to a two-week time step. The actual (nontransformed) $P$-values are reported below the bars. The dashed line represents the 0.05 threshold. 


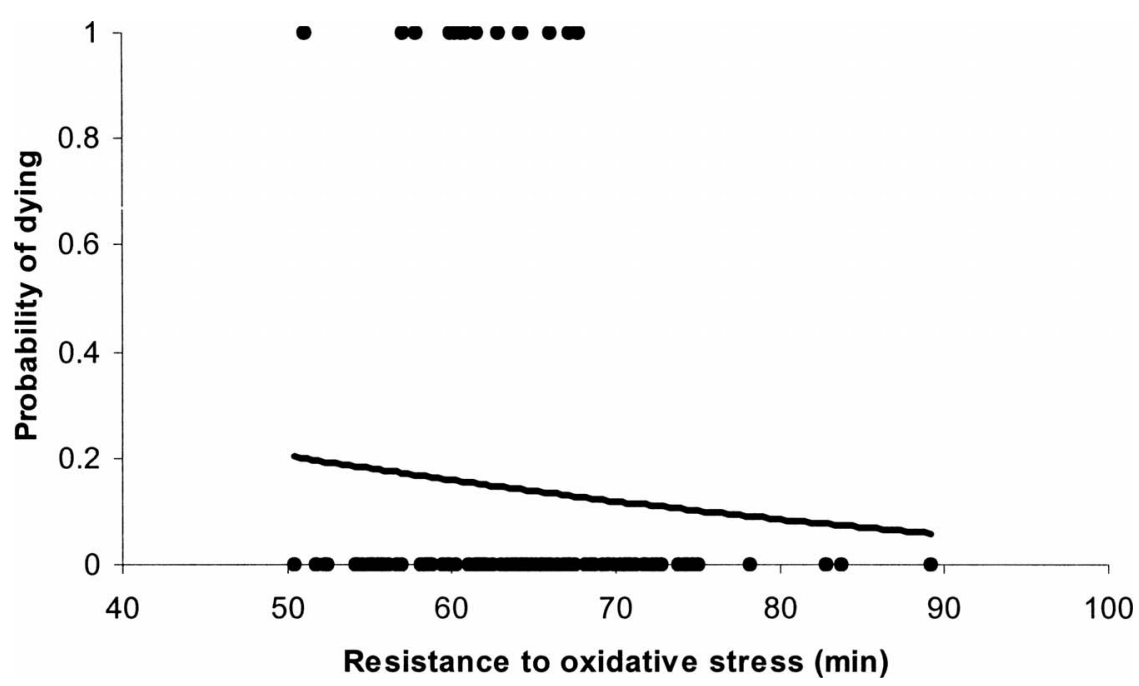

FIG. 6. Resistance to oxidative stress and mortality risk. The figure shows the relationship between the resistance to oxidative stress assessed on September 19 and the likelihood of dying in the following three months. The dots represent the fate of individuals $(1=$ dead, $0=$ alive). The fitted line has been obtained from a generalized linear mixed model with binomial error and logit link (GLIMMIX), taking into account the identity of original and foster nests as random factors.

but also in shorter life span, although the experimental brood size did not directly affect any of these life-history traits (number of breeding events, number of fledglings produced and longevity). Although the population growth rate of birds from reduced broods was higher than that of birds from enlarged broods (a 3.46-fold increase), this difference was not statistically significant. However, the pattern of age-specific decline in reproductive value significantly differed between the two brood size groups, suggesting that birds from reduced broods had an accelerated senescence. Finally, resistance to oxidative stress varied with age, predicted short-term mortality and was negatively correlated with breeding effort, although the correlation between resistance to oxidative stress and longevity was not statistically significant. These results are in agreement with some of the predictions of the disposable-soma theory of the evolution of aging and suggest that antioxidant defenses could be a key resource that might account, at the proximate level, for the deterioration of performance with age.

There is now growing evidence showing that early condition affects more or less permanently a number of phenotypic traits (Metcalfe and Monaghan 2001). Undernutrition, both at the prenatal and postnatal level, is associated with several physiological syndromes (Patel and Srinivasa 2002; Bateson et al. 2004), and with reduced adult body size (De Kogel 1997), fecundity and survival (Sayer and Cooper 2002; Lumma 2003; Ozanne and Hales 2004). By manipulating brood size, we indirectly manipulated the nutritional status of the nestlings (Deerenberg et al. 1996; De Kogel 1997). In agreement with previous findings, brood size manipulation resulted in smaller and leaner nestlings at the age of 20 days. However, this difference tended to decrease at the age of 60 days and completely disappeared for breeding birds (September sampling event: $F_{1,41.3}=0.72, P=0.401$, mean \pm SE: $15.33 \pm 0.29$ and $14.96 \pm 0.34 \mathrm{~g}$, for reduced and enlarged broods, respectively), suggesting that a compensatory growth took place (see also Birkhead et al. 1999).
Beyond the obvious effect on growth, early environmental conditions affected a number of physiological parameters such as cell-mediated immune response and plasma carotenoids. These effects can of course also reflect the reduced availability of energy/resources suffered by enlarged-brood nestlings. The importance of early environmental conditions on immunity (Sorci et al. 1997), and antioxidant defenses (Robles et al. 2001; Blount et al. 2003) has already been emphasized in previous studies. For instance, in humans, preterm neonates show increased susceptibility to oxidative stress and reduced levels of antioxidant defenses in the first days of life compared to full term neonates (Robles et al. 2001). This difference is thought to be generated by the incomplete transfer of nutrients with antioxidant properties during the final stage of the gestation and the time needed by the antioxidant enzymatic system to fully mature (Robles et al. 2001, see also Boda et al. 1998; Brzezinska-Slebodzinska 2001). A recent study on captive zebra finches showed that chicks fed with poor-quality food during their first weeks of life have lower blood levels of antioxidant molecules (i.e., vitamin $\mathrm{A}$, vitamin $\mathrm{E}$ and carotenoids) when becoming adults, compared to chicks fed with a standard diet (Blount et al. 2003). These results were explained as the consequence of differences in diet composition (i.e., proportion of protein and antioxidants), which would have affected the synthesis of lipoproteins necessary for the uptake and carrying of lipophilic antioxidants (i.e., Nutting et al. 2002). This explanation could also apply to the results reported in our study.

The effect of manipulating nestling competition was not limited to the prematurational physiology. Individuals raised in enlarged broods reproduced later than birds from reduced broods. The onset of breeding appeared to be the key lifehistory trait because it was correlated with the other reproductive parameters, early breeding birds reproducing more and fledging more offspring. Natural selection is generally expected to favor early breeding individuals because of their higher chances of surviving to maturity (Cole 1954). This is 

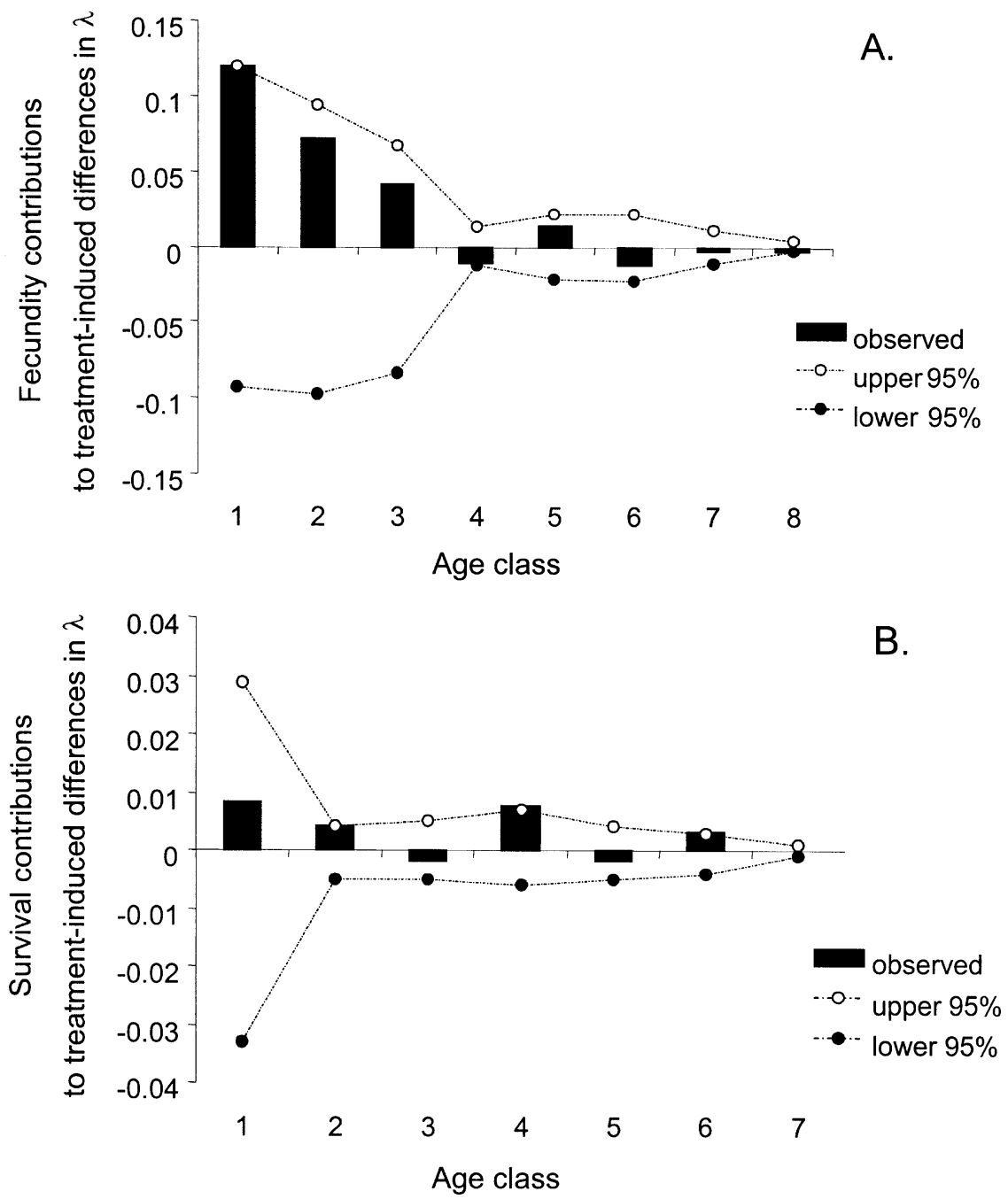

FIG. 7. Contributions of differences in age-specific fecundity (A) and survival (B) to the effect of the experimental brood size on population growth rate $(\lambda)$. Empty and full dots represent the upper $95 \%$ and lower $5 \%$ percentile distribution of age-specific contributions for 500 permuted data sets. For details, see life table response experiment modeling in Materials and Methods.

of course particularly true for organisms with high extrinsic mortality rate (Stearns 1992), such as wild populations of zebra finches (Zann 1996). Interestingly, even in the absence of two of the main sources of extrinsic mortality (i.e., predation and starvation), as experienced by our captive zebra finches, early breeders had a considerable selective advantage, as witnessed by their higher lifetime reproductive success. In agreement with the idea of strong selection pressures acting on age at first reproduction in this species, zebra finches have a particularly precocious breeding age, even compared to other phylogenetically related species (Zann 1996). Therefore, it is likely that the fitness consequences of our brood size manipulation could have been even more pronounced in the wild, where very few birds survive long enough to experience two breeding periods (Zann 1996).

By following the cohort of birds throughout their entire life span we were able to address some specific predictions issued from the disposable-soma theory of aging. The disposable-soma theory postulates that investment in the maintenance of somatic tissue should not exceed the level required to maintain the body functions through the normal expectation of life span, under the predominant regime of extrinsic mortality (Kirkwood 2005). Any extra investment in durability would not be beneficial for two reasons: first, it would not prove effective because of the increasing chance of accidental death; second, it would divert resources from reproduction. Specifically, the disposable-soma theory predicts that (1) aging results from the accumulation of unrepaired damages due to limitations in somatic maintenance and repair; (2) limitations in somatic maintenance arise from the allocation of metabolic resources to reproduction; (3) the allocation of resources to maintenance is determined by evolutionary optimization and the allocation strategy should be plastic to respond to environmental variation (Kirkwood 2005). In agreement with these predictions we found that: (1) although resistance to oxidative stress was not correlated with life span, it showed a parabolic function with age and predicted short-term mortality (up to three months after the assessment of resistance to oxidative stress); (2) individuals that invested more in reproduction, assessed as the number 

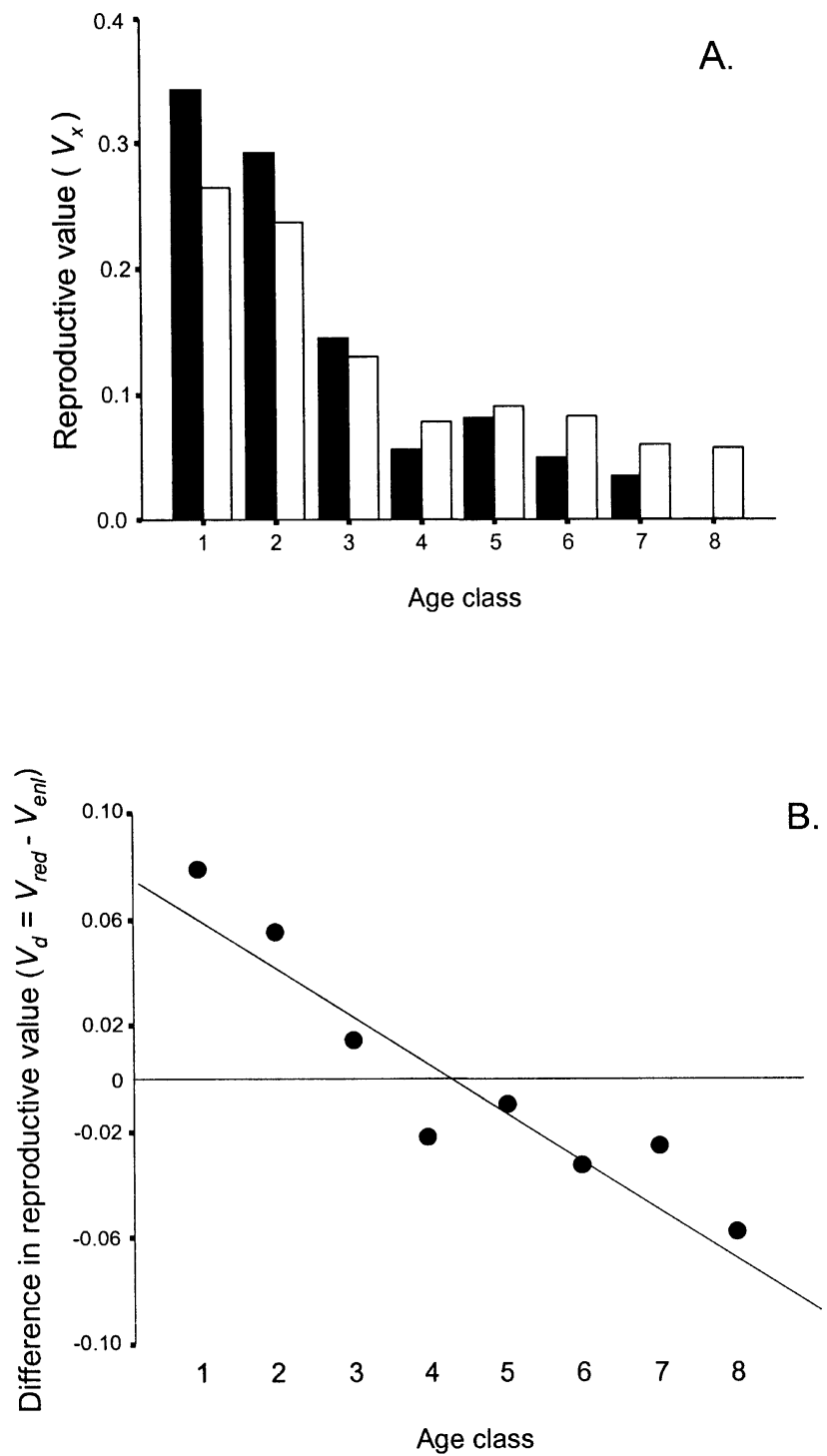

FIG. 8. Birds raised in reduced broods showed a greater decrease in reproductive value with age than individuals raised in enlarged broods. (A) Reproductive values ( $v$ ) for reduced (black bars) and enlarged (white bars) broods as a function of age. (B) Differences in reproductive values $\left(v_{d}\right)$ of birds issued from reduced and enlarged broods as a function of age. The pattern was best described by a linear regression with a standardized slope $=-0.934$. Only seven of 500 randomly generated slopes were lower than the observed one $(P=0.014)$.

of total reproductive attempts, had a weaker resistance to oxidative stress; and (3) the reproductive value of birds issued from reduced broods decreased with age more sharply than the reproductive value of birds from enlarged broods.

The role played by ROS as important proximate contributors to the aging process has been repeatedly emphasized (Sohal and Weindruch 1996; Finkel and Holbrook 2000; Melov et al. 2000). Reactive oxygen species are very reactive molecules with harmful effects on cell structures (Finkel and Holbrook 2000). Allocation of resources to antioxidant defenses is therefore important to maintain homeostasis (Finkel and Holbrook 2000). Recent experimental evidence suggests that enhanced reproductive effort reduces the antioxidant defenses in both fruit flies (Salmon et al. 2001; Wang et al. 2001) and zebra finches (Alonso-Alvarez et al. 2004b; Wiersma et al. 2004). In agreement with these previous findings, we found that, whereas birds from enlarged broods presented reduced resistance against a free radical attack when 60 days old, this pattern was reversed after a period of intense reproductive effort. Although suggestive, we should keep in mind that these are correlative results and a more rigorous experimental design to test this idea would be to compare birds that are allowed to breed versus birds that are prevented from breeding.

In addition to the analyses based on individual values, we also adopted a population-based approach: the life table response experiment modeling. The rationale of the LTRE is that a treatment (in our case the experimental brood size) can affect various vital rates in an age-specific manner. Projection matrices provide the tool to combine the effect of the treatment into synthetic parameters, such as population growth rate, or reproductive value. Reproductive value is a particularly interesting parameter in the context of the present study because it represents the prospects for future reproduction viewed at successive ages after birth (Fisher 1930; Partridge and Mangel 1999). Reproduction, survival, and timing all contribute to reproductive value and it has been suggested that the change in reproductive value with age is the appropriate measure of the rate of aging (Newton and Rothery 1997; Partridge and Mangel 1999). We found that early modification of environmental conditions, due to the manipulated brood size, significantly changed the pattern of decrease of reproductive value with age. Birds from reduced broods had a sharper decline in reproductive value than birds from enlarged broods. This result shows that apparently reversible environmental conditions can have a profound impact on the life-history trajectories and stresses the idea that allocation strategies to reproduction vs. maintenance can respond in a plastic manner to environmental changes.

\section{ACKNOWLEDGMENTS}

We thank the staff of the Station Biologique de Foljuif (École Normale Supérieure). Financial support was provided by the Ministère de la Recherche (Action Concertée Incitative Jeunes Chercheurs to GS), and the Université de Bourgogne (Bonus Qualité Recherche to GD, JP and BF). CA-A was consectively supported by the Parga Pondal and the Ramón y Cajal Fellowships (Xunta de Galacia and Spanish Ministerio de Educación y Ciencia, respectively).

\section{Literature Cited}

Alonso-Alvarez, C., and J. L. Tella. 2001. Effects of experimental food restriction and body-mass changes on avian T-cell mediated immune response. Can. J. Zool. 79:101-105.

Alonso-Alvarez, C., S. Bertrand, G. Devevey, M. Gaillard, J. Prost, B. Faivre, and G. Sorci. 2004a. An experimental test of the dosedependent effect of carotenoids and immune activation on sexual signals and antioxidant activity. Am. Nat. 164:651-659.

Alonso-Alvarez, C., S. Bertrand, G. Devevey, J. Prost, B. Faivre, and G. Sorci. 2004b. Oxidative stress as a proximate cost of reproduction. Ecol. Lett. 7:363-368.

Bateson, P., D. Barker, T. Clutton-Brock, D. Deb, B. D’Udine, R. A. Foley, P. Gluckman, K. Godfrey, T. Kirkwood, M. Mirazón 
Lahr, J. McNamara, N. B. Metcalfe, P. Monaghan, H. G. Spencer, and S. E. Sultan. 2004. Developmental plasticity and human health. Nature 430:419-421.

Bertrand, S., C. Alonso-Alvarez, G. Devevey, B. Faivre, J. Prost, and G. Sorci. 2006. Carotenoids modulate the trade-off between egg production and resistance to oxidative stress in zebra finches. Oecologia 147:576-584.

Birkhead, T. R., F. Fletcher, and E. J. Pellatt. 1999. Nestling diet, secondary sexual traits and fitness in the zebra finch. Proc. R. Soc. Lond. B 266:385-390.

Blache, D., and M. Prost. 1992. Free radical attack: biological test for human resistance capability. Pp. 82-98 in G. Ponnamperuma, and C. W. Gehrke, eds. A lunar-based chemical analysis laboratory. Deepak A, Hampton, VA.

Blount, J. D., N. B. Metcalfe, K. E. Arnold, P. F. Surai, G. L. Devevey, and P. Monaghan. 2003. Neonatal nutrition, adult antioxidant defences, and sexual attractiveness in the zebra finch. Proc. R. Soc. Lond. B 270:1691-1696.

Boda, V., B. Finckh, M. Dürken, J. Commentz, H.-H. Hellwege, and A. Kohlschütter. 1998. Monitoring erythrocyte free radical resistance in neonatal blood microsamples using a peroxyl radical-mediated haemolysis test. Scand. J. Clin. Lab. Inv. 58: 317-322.

Brown, M. E. 1996. Assessing body condition in birds. Pp. 67-135 in V. Nolan and E. D. Ketterson, eds. Current ornithology, Volume 13. Plenum Press, New York.

Brzezinska-Slebodzinska, E. 2001. Erythrocyte osmotic fragility test as the measure of defence against free radicals in rabbits of different age. Acta Vet. Hung. 49:413-419.

Burley, N. 1988. Wild zebra finches have band-color preferences. Anim. Behav. 36:1235-1237.

Carranza, J., S. Alarcos, C. B. Sánchez-Prieto, J. Valencia, and C. Mateos. 2004. Disposable-soma senescence mediated by sexual selection in an ungulate. Nature 432:215-218.

Caswell, H. 2001. Matrix population models. Construction, analysis, and interpretation. 2d ed. Sinauer Associates, Sunderland, MA.

Cole, L. C. 1954. The population consequences of life history phenomena. Q. Rev. Biol. 29:103-137.

Dawson, R. D., and G. R. Bortolotti. 1997. Total plasma protein level as an indicator of condition in wild American kestrels (Falco sparverius). Can. J. Zool. 75:680-686.

Deerenberg, C., C. H. De Kogel, and G. F. J. Overkamp. 1996. Costs of reproduction in the zebra finch Taeniopygia guttata: manipulation of brood size in the laboratory. J. Avian Biol. 27: 321-326.

De Kogel, C. H. 1997. Long-term effects of brood size manipulation on morphological development and sex-specific mortality of offspring. J. Anim. Ecol. 66:167-178.

Finkel, T., and N. J. Holbrook. 2000. Oxidants, oxidative stress, and the biology of ageing. Nature 408:239-247.

Fisher, R. A. 1930. The genetical theory of natural selection. Dover, New York

Girard, A., S. Madani, E. S. El Boustani, J. Belleville, and J. Prost. 2005. Changes in lipid metabolism and antioxidant defense status in spontaneously hypertensive rats and Wistar rats fed a diet enriched with fructose and saturated fatty acids. Nutrition 21: 240-248.

Girodon, F., D. Blache, A. L. Monget, M. Lombart, P. BrunetLecompte, J. Arnaud, M. J. Richard, and P. Galan. 1997. Effect of a two-year supplementation with low doses of antioxidant vitamins and/or minerals in elderly subjects on levels of nutrients and antioxidant defense parameters. J. Am. Coll. Nutr. 16: $357-365$.

Gluckman, P. D., M. A. Hanson, H. G. Spencer, and P. Bateson. 2005. Environmental influences during development and their later consequences for health and disease: implications for the interpretation of empirical studies. Proc. R. Soc. Lond. B 272: 671-677.

Gonzalez, G., G. Sorci, A. P. Møller, P. Ninni, C. Haussy, and F. de Lope. 1999. Immunocompetence and condition-dependent sexual advertisement in male house sparrows (Passer domesticus). J. Anim. Ecol. 68:1225-1234.
Goto, N., H. Kodama, K. Okada, and Y. Fujimoto. 1978. Suppression of phytohemagglutinin skin response in thymectomized chickens. Poult. Sci. 57:246-250.

Harman, D. 1957. Aging: a theory based on free radical and radiation chemistry. J. Gerontol. 2:298-300.

Kirkwood, T. B. L. 1977. Evolution of ageing. Nature 270:301-304. . 2005. Understanding the odd science of aging. Cell 120: 437-447.

Kirkwood, T. B. L., and M. R. Rose. 1991. Evolution of senescence: late survival sacrificed for reproduction. Philos. Trans. R. Soc. Lond. B Biol. Sci. 332:15-24.

Lesgards, J. F., P. Durand, M. Lassarre, P. Stocker, G. Lesgards, A. Lanteaume, M. Prost, and M.-P. Lehucher-Michel. 2002. Assessment of lifestyle effects on the overall antioxidant capacity of healthy subjects. Environ. Health Perspect. 110:479-487.

Levin, L., H. Caswell, T. Bridges, C. DiBacco, D. Cabrera, and G. Plaia. 1996. Demographic responses of estuarine polychaetes to pollutants: life table response experiments. Ecol. Appl. 6: 1295-1313.

Lindström, J. 1999. Early development and fitness in birds and mammals. Trends Ecol. Evol. 14:343-348.

Littell, R. C., G. A. Milliken, W. W. Stroup, and R. D. Wolfinger. 1996. SAS system for mixed models. SAS Institute, Cary, NC.

Lumma, V. 2003. Early development conditions and reproductive success in humans: downstream effects of prenatal famine, birth weight, and timing of birth. Am. J. Human Biol. 15:370-379.

Lumma, V., and T. Clutton-Brock. 2002. Early development, survival and reproduction in humans. Trends Ecol. Evol. 17: 141-147.

Melov, S., J. Ravenscroft, S. Malik, M. S. Gill, D. W. Walker, P. E. Clayton, D. C. Wallace, B. Malfroy, S. R. Doctrow, and G. J. Lithgow. 2000. Extension of life-span with superoxide dismutase/catalase mimetics. Science 289:1567-1569.

Metcalfe, N. B., and P. Monaghan. 2001. Compensation for a bad start: grow now, pay later? Trends Ecol. Evol. 16:254-260.

Newton, I., and P. Rothery. 1997. Senescence and reproductive value in sparrowhawks. Ecology 78:1000-1008.

Nutting, D. F., N. S. Kumar, S. A. Siddiqi, and C. M. Mansbach 2002. Nutrient absorption. Curr. Opin. Gasteroenterol. 18: $168-175$.

Ozanne, S. E., and C. N. Hales. 2004. Catch-up growth and obesity in male mice. Nature 427:411-412.

Partridge, L., and M. Mangel. 1999. Messages from mortality: the evolution of death rates in the old. Trends Ecol. Evol. 14 438-442.

Patel, M. S., and M. Srinivasa. 2002. Metabolic programming: Causes and consequences. J. Biol. Chem. 277:1629-1632.

Pieri, C., F. Moroni, and M. Marra. 1996. Food restriction increases the protection of erythrocytes against the hemolysis induced by peroxyl radicals. Mech. Aging Dev. 87:15-23.

Reznick, D. 1992. Measuring the costs of reproduction. Trends Ecol. Evol. 7:42-45.

Robles, R., N. Palomino, and A. Robles. 2001. Oxidative stress in the neonate. Early Hum. Dev. 65:S75-S81.

Rojas Wahl, R. U., L. Zeng, S. A. Madison, R. L. DePinto, and B. J. Shay. 1998. Mechanistic studies on the decomposition of water soluble azo-radical-initiators. J. Chem. Soc., Perk. T. 29: 2009-2018.

Rose, M. R., and T. J. Bradley. 1998. Evolutionary physiology of the cost of reproduction. Oikos 83:443-451.

Salmon, A. D., D. B. Marx, and L. G. Harshman. 2001. A cost of reproduction in Drosophila melanogaster: stress susceptibility. Evolution 55:1600-1608.

SAS Institute. 2001. SAS/STAT software: Changes and Enhancements, Version 8.2. SAS Publishing, Cary, NC.

Sayer, A. A., and C. Cooper. 2002. Early diet and growth: impact on ageing. Proc. Nutr. Soc. 61:79-85.

Smits, J. E., G. R. Bortolotti, and J. L. Tella. 1999. Simplifying the phytohemagglutinin skin testing technique in studies of avian immunocompetence. Funct. Ecol. 13:567-572.

Sohal, R. S., and R. Weindruch. 1996. Oxidative stress, caloric restriction, and aging. Science 273:59-63.

Sorci, G., J. J. Soler, and A. P. Møller. 1997. Reduced immuno- 
competence of nestlings in replacement clutches of the European magpie (Pica pica). Proc. R. Soc. Lond. B 264:1593-1598.

Stearns, S. C. 1992. The evolution of life histories. Oxford Univ. Press, Oxford, U.K.

Stocker, P., J. -F. Lesgards, N. Vidal, F. Chalier, and M. Prost. 2003. ESR study of a biological assay on whole blood: antioxidant efficiency of various vitamins. Bioch. Bioph. Acta 1621: $1-8$.

von Schantz, T. V., S. Bensch, M. Grahn, D. Hasselquist, and H. Wittzell. 1999. Good genes oxidative stress and condition-dependent sexual signals. Proc. R. Soc. Lond. B 266:1-12.

Wang, Y., A. B. Salmon, and L. G. Harshman. 2001. A cost of reproduction: oxidative stress susceptibility is associated with increased egg production in Drosophila melanogaster. Exp. Gerontol. 36:1349-1359.

Wiersma, P., C. Selman, J. R. Speakman, and S. Verhulst. 2004. Birds sacrifice oxidative protection for reproduction. Proc. R. Soc. Lond. B, Biol. Lett. Suppl. 5:S360-S363.

Zann, R. A. 1996. The zebra finch. A synthesis of field and laboratory studies. Oxford Univ. Press, Oxford, U.K.

Zera, A. J., and L. G. Harshman. 2001. The physiology of life history trade-offs in animals. Annu. Rev. Ecol. Syst. 32:95-126.

Corresponding Editor: D. Promislow 